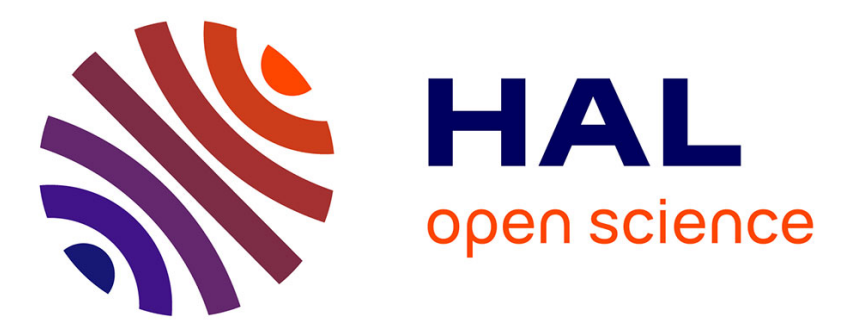

\title{
On-Surface Atom-by-Atom-Assembled Aluminum Binuclear Tetrabenzophenazine Organometallic Magnetic Complex
}

We -Hyo Soe, We -Hyo Soe, Carlos Manzano, Roberto Robles, Nicolas Lorente, Christian Joachim

\section{To cite this version:}

We -Hyo Soe, We -Hyo Soe, Carlos Manzano, Roberto Robles, Nicolas Lorente, et al.. On-Surface Atom-by-Atom-Assembled Aluminum Binuclear Tetrabenzophenazine Organometallic Magnetic Complex. Nano Letters, 2019, 20 (1), pp.384-388. 10.1021/acs.nanolett.9b04040 . hal-03004565

\section{HAL Id: hal-03004565 https://hal.science/hal-03004565}

Submitted on 13 Nov 2020

HAL is a multi-disciplinary open access archive for the deposit and dissemination of scientific research documents, whether they are published or not. The documents may come from teaching and research institutions in France or abroad, or from public or private research centers.
L'archive ouverte pluridisciplinaire HAL, est destinée au dépôt et à la diffusion de documents scientifiques de niveau recherche, publiés ou non, émanant des établissements d'enseignement et de recherche français ou étrangers, des laboratoires publics ou privés. 


\section{On-Surface Atom by Atom Assembled Aluminium}

\section{Bi-Nuclear Tetrabenzophenazine Organo-Metallic}

\section{Magnetic Complex}

We-Hyo Soe, ${ }^{* \dagger}$ Carlos Manzano, ${ }^{*}$ Roberto Robles, ${ }^{\S}$ Nicolas Lorente ${ }^{\S}$ and

Christian Joachim ${ }^{\dagger}$

${ }^{\dagger}$ Centre d'Elaboration de Matériaux et d’Études Structurales (CEMES), Centre National de la Recherche Scientifique (CNRS), Université de Toulouse, 29 Rue J. Marvig, BP 4347, 31055

Toulouse Cedex, France

Institute of Material Research and Engineering (IMRE), Agency for Science, Technology and Engineering (A*STAR), 3 Research Link, Singapore, 117602, Singapore

${ }^{\S}$ Centro de Física de Materiales CFM/MPC (CSIC-UPV/EHU), Paseo Manuel de Lardizabal 5, 20018 Donostia-San Sebastián, Spain

KEYWORDS: Single molecule magnet, Kondo resonance, Aluminum-organic molecule complex, LT-UHV STM, Molecule manipulation. 
ABSTRACT: The Kondo effect results from the interactions of the conduction electrons in a metal bulk with localized magnetic impurities. ${ }^{1,2}$ While adsorbed atop a metallic surface, the on-surface nanoscale version of this effect is observed when a single magnetic atom ${ }^{3}$ or a single magnetic molecule $(\mathrm{SMM})^{4,5}$ is interacting with the conduction electrons. SMM are commonly organometallic complex incorporating transition metal atoms in different oxidation states. ${ }^{6-8} \mathrm{We}$ demonstrate how a single non-magnetic neutral tetrabenzo[a,c,j,h]phenazine molecule can be onsurface coordinated with exactly two aluminum metal atoms ( $\mathrm{Al}(\mathrm{III})$ oxidation state on the $\mathrm{Au}(111)$ surface) by low-temperature scanning tunneling microscope (LT-STM) single atom manipulation. It results a Kondo measurable localized molecular magnetic moment. This opens a new way to design SMM complex without the need of heavy transition metal atom and complex ligand to stabilize the molecular coordination sphere.

TOC

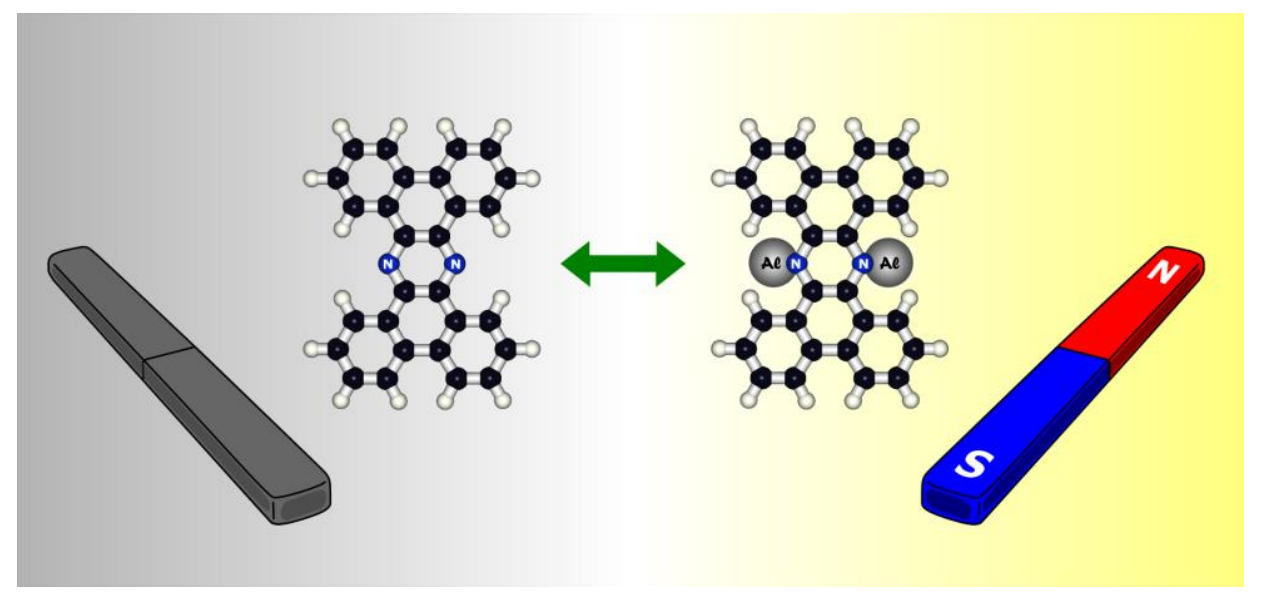


On an $\mathrm{Au}(111)$ surface, single $\mathrm{Al}_{2}$-tetrabenzo[a,c,j,h]phenazine organo-metallic complexes were constructed using aluminium atom by atom LT-STM single atom/molecule manipulation protocols. The Kondo effect was characterized by measuring the local differential conductance (dI/dV) scanning tunneling spectra (STS). DFT calculations confirm the stabilization of the $\mathrm{Al}_{2}-$ complex magnetic moment on the $\mathrm{Au}(111)$ surface. Figure 1(b) is presenting the LT-UHV STM constant current topographic image of tetrabenzo[a,c,j,h]phenazine molecules co-adsorbed with single $\mathrm{Al}$ atoms on an $\mathrm{Au}(111)$ metallic surface. The molecules (chemical structure Fig. 1(a)) are imaged having the shape of an ' $\mathrm{H}$ ' letter. Single $\mathrm{Al}$ ad-atoms are clearly identified as well as the underlying herringbone reconstruction of the $\mathrm{Au}(111)$ surface. The two $\mathrm{Al}$ atoms are coordinated by manipulating the H-molecule towards those Al's using the STM in a pushing manipulation mode forming the $\mathrm{Al}_{2}$ organo-metallic complex (Ref. 9. ${ }^{9}$ ) A single $\mathrm{Al}$ atom invariably binds to one nitrogen of the central pyrazine ring as anticipated by design. Attempts by STM manipulations to coordinate an $\mathrm{Al}$ atom on other parts of the $\mathrm{H}$-molecule result in the $\mathrm{Al}$ atom always binding to one of those nitrogen's central pyrazine ring. This on-surface coordination experiment was also attempted with a single Au atom. As expected, an Au atom manipulated towards the pyrazine central ring is showing a lower affinity with the nitrogen's as compared to Al. It always settled underneath one of the four off-center phenyl rings (see Suppl. Info. S1).

Once a single $\mathrm{Al}$ is on-surface complexed with one of the 2 available central nitrogens of the $\mathrm{H}$ molecule (see below for discussion), the central part of this $\mathrm{Al}_{1}$-complex is STM imaged with a brighter contrast than the center of a bare molecule as presented in Fig. 1(d). After a second STM tip molecule manipulation, another $\mathrm{Al}$ can be coordinated to the remaining free central nitrogen leading to an on-surface $\mathrm{Al}_{2}$-molecule complex not stable in solution. STM imaged, this new 
complex presents an even brighter central contrast than the $\mathrm{Al}_{1}$-complex one as presented in Fig. $1(\mathrm{e})$.

The detailed electronic structures of the bare $\mathrm{H}$-molecule, of the $\mathrm{Al}_{1}$ - and $\mathrm{Al}_{2}$-complexes were characterized using local STS dI/dV measurements completed by DFT calculations. When the STM tip is located between two corner phenyls and near one of the nitrogen sites of the bare molecule, three distinct tunneling electronic resonances are observed. In first approximation, they can be attributed to the H-molecule $\mid \mathrm{S}^{+}>$(hole) and $\mid \mathrm{S}^{-}>$(electron) tunneling resonances of the molecular electronic $|\mathrm{S} 0\rangle$ ground and $\mid \mathrm{S} 1>$ first excited singlet states respectively. They are captured at applied voltages higher than $1.5 \mathrm{~V}$ on each polarity (See the STS spectrum in Figure 2(a)). When the tip is located at the center of the bare molecule, those resonances are not existing in the $[-1.0 \mathrm{~V},+1.0 \mathrm{~V}]$ narrow voltage window as also presented in Fig. 2(b). Here, the STS spectra recorded on an $\mathrm{Au}(111)$ terrace (black line) and on this molecule (red) are identical.

In this $[-1.0 \mathrm{~V},+1.0 \mathrm{~V}]$ bias voltage interval, the $\mathrm{STS}$ spectrum recorded on the $\mathrm{Al}_{1}$-molecule complex demonstrates that the $\mathrm{Au}(111)$ surface state was smoothed and shifted below its usual $500 \mathrm{mV}$ energy location. This is because the single $\mathrm{Al}$ atom of the $\mathrm{Al}_{1}$-complex is a strong scattering center for the surface electron standing waves nearby the $\mathrm{Al}_{1}$-molecule complex center ${ }^{10}$ as presented in Fig. 2(b) by the blue STS spectrum. Another distinctive feature of this tunneling spectrum is a notable increase of the differential conductance for a positive bias voltage coming from a down shift of the $+1.9 \mathrm{~V}$ Fig. 2(a) $\mid \mathrm{S1}^{-}>$tunneling resonance. New Ref. A ${ }^{\text {ref }}$

After the construction of the $\mathrm{Al}_{2}$-molecule complex by molecule manipulation and as presented by the Fig. 2(b) green spectrum, a new very sharp peak is recorded exactly at a zero bias voltage. 
According to its sharp symmetric shape, this is the signature of a Kondo resonance with a Kondo temperature of $30.3 \mathrm{~K}$ (see Suppl. Info. S2) indicating that the $\mathrm{Al}_{2}$-molecule complex is magnetic on the Au(111) surface (new Ref. B R. Zitko PRB 84, 195116 (2011)). ${ }^{11}$

Self-consistent field (SCF) DFT calculations were performed with one H-molecule per unit cell on the $\mathrm{Au}(111)$ surface (see Methods). With an adsorption energy of $3.38 \mathrm{eV}$, the $\mathrm{H}$-molecule adsorbs flat on $\mathrm{Au}(111)$ with its central pyrazine stabilized on a bridge site. For this lowest-energy surface conformation, each pair of terminal phenyl rings is matching with two Au top surface atoms (see Suppl. Info. Fig. S3(a)). When forcing the pyrazine to adsorb atop an Au site, the adsorption energy increase only by $29 \mathrm{meV}$ indicating a very low lateral diffusion barrier on $\mathrm{Au}(111)$. Using a Bader analysis, there is only a small 0.16 electrons small donation to the surface and the H-molecule HOMO-LUMO gap (highest occupied - lowest unoccupied molecular orbitals) is centered around the $\mathrm{Au}(111)$ Fermi level. Notice that HOMO and LUMO are the main monoelectronic states entering respectively in the composition of the $\mid \mathrm{S0}^{+}>$and $\mid \mathrm{S}^{-}>$quantum states required to describe the recorded electronic resonances in Fig. 2(a).

One and two Al (respectively $\mathrm{Au}$ ) single ad-atoms were also adsorbed in the unit cell (see Methods). When one $\mathrm{Al}$ ad-atom is located next to the phenanthrazine molecule, it stabilizes underneath, next to a nitrogen and on a bridge site. The molecule minimizes its surface energy by approaching the $\mathrm{Al}$ site. It shifts its central pyrazine slightly away from its original bridge site (see Fig. S3(b)). During this on-surface stabilization, the H-molecule captures a tiny amount of charge (0.04 electrons). This charge transfer induces a shift down of the LUMO energy in $\mid \mathrm{S}^{-}>$state as 
shown in Fig. 2(c). Altogether, the Al atom had lost 1.86 electrons mainly transferred to the $\mathrm{Au}(111)$ surface without developing a local magnetic moment in the $\mathrm{Al}_{1}$-molecule complex.

Adding the second $\mathrm{Al}$ on the central pyrazine leads to a stabilization of the $\mathrm{Al}_{2}$-molecule complex with its shift to a hollow site (see Fig. S3(c)). As compared with the $\mathrm{Al}_{1}$-complex, the coordination of two Al triggers a large electronic structure transformation. Each Al ad-atom has now lost about two electrons and one electron was transferred to the H-molecule explaining the observed molecular local magnetic moment leading to the zero voltage Kondo resonance. The SCF value of this magnetic moment is 0.41 Bohr magnetons with a filling of about 0.65 electrons spinup and 0.24 electrons spin-down at the Fermi energy.

The main oxidation state of aluminum is well-known and defined as $\mathrm{Al}(\mathrm{III})$. When $\mathrm{Al}$ is chemisorbed on the metal surface, this oxidation number is no more valid. For a single isolated $\mathrm{Al}$ chemisorbed on $\mathrm{Au}(111)$, DFT calculations give an oxidation state between $\mathrm{Al}(\mathrm{I})$ and $\mathrm{Al}(\mathrm{II})$ and low-valent aluminum compounds had been already obtained in solution. ${ }^{12}$ About 1.82 electrons are missing from the $\mathrm{Al} 3 \mathrm{p}$ and the $3 \mathrm{~s}$ native orbitals. This intermediate oxidation state beneficiates to the observed coordination between the neutral $\mathrm{H}$-molecule and the 2 STM manipulated Al atoms. Notice that in solution, the closest known compound is $\left(\mathrm{Al}(\text { Methyl })_{3}\right)_{2}$-pyrazine. ${ }^{13}$ On the $\mathrm{Au}(111)$ surface, the consequence of this intermediate oxidation state is that the $\mathrm{Al}_{2}$-molecule complex is magnetic as probed using the Kondo effect. Indeed, the analysis of the spin distribution on the $\mathrm{Al}_{2}$-complex (see Fig. 2c and Fig. 3(d)) indicates that its central pyrazine is spin polarized. To understand this distribution, we have calculated different $\mathrm{Al}_{2}$-molecule complex oxidation states away from its stabilizing surface while keeping its surface optimized conformation. We first considered $\mathrm{Al}_{2}$-molecule complex with two neutral $\mathrm{Al}$ atoms. Far away from the surface, this 
artificial molecule has no magnetic moment. As indicated by our charge analysis of the $\mathrm{Al}_{2}$ molecule complex on the $\mathrm{Au}(111)$ surface, removing three electrons from this $\mathrm{Al}_{2}$-complex produces a large spin polarization but not comparable with the adsorbed complex one (Fig. 3(e)). However, the spin distribution of a H-molecule anion alone (Fig. 3(f)) is showing a very good agreement with the adsorbed $\mathrm{Al}_{2}$-molecule complex experimental Kondo map Fig. 3b. This confirms that the $2 \mathrm{Al}$ atoms are partially charging negatively the $\mathrm{H}$-molecule because of their adsorption on the $\mathrm{Au}(111)$ surface. They serve as spin donor for the molecule electronic cloud. The advantage of this on-surface coordination chemistry is that the surface $\mathrm{Al}_{2}$-molecule constructed complex is magnetic as compared with the $\left(\mathrm{Al}(\text { Methyl })_{3}\right)_{2}$-pyrazine molecule. ${ }^{13}$

Using Au ad-atoms on the $\mathrm{Au}(111)$ surface do not lead to a spin polarization of the H-molecule. The molecule-to-surface charge transfer is slightly increasing ( 0.24 electrons for two Au ad-atoms) to the molecule instead of the electron transfer in the case of $\mathrm{Al}$ ad-atoms. This can be rationalized observing that $\mathrm{Au}$ ad-atoms do not change locally the $\mathrm{Au}(111)$ work function and the local electrostatic balance on the surface.

\section{CONCLUSION}

Light metallic ad-atoms can be on-surface coordinated to partially charge a neutral organic molecule on a noble metal surface, spin polarize this molecule and create an on-surface single molecule magnet organo-metallic complex. We have used exactly two $\mathrm{Al}$ atoms pre-positioned on an $\mathrm{Au}(111)$ surface. At a zero bias voltage, a Kondo resonance with a temperature of $30.3 \mathrm{~K}$ was STS measured. The magnetism of our $\mathrm{Al}_{2}$-tetrabenzo[a,c,j,h]phenazine complex rationalizes by comparing the electron affinity and ionization energies of the different partners involved: the 
surface, the $\mathrm{Al}$ ad-atom and the neutral molecule. The $\mathrm{Au}(111)$ surface has a work function larger than the H-molecular affinity and smaller than its ionization energy. As a consequence, almost no charge transfer takes place between the molecule and the surface. With its small oxidation energy, $\mathrm{Al}$ ad-atoms are efficiently transferring electrons to the $\mathrm{Au}(111)$ surface and the molecule becomes spin polarized. On a surface and having the possibility to use light metal atoms with a neutral small organic molecule open the way to design new and light organo-metallic magnetic molecular materials.

\section{Methods}

Tetrabenzo[a,c,j,h]phenazine molecules were sublimated using free evaporation by heating a quartz crucible at $240^{\circ} \mathrm{C}$. The evaporation parameters were selected to deposit a minute amount of molecules to produce a sub-monolayer coverage to avoid molecules from clustering and to provide enough space in between the deposited Al atoms and molecules to perform the STM molecule manipulations. The H-molecules were deposited on an $\mathrm{Au}(111)$ mono-crystal which was cleaned using standard methods consisting of several cycles of Ar+ ion sputtering and subsequent annealing. During the molecules deposition, the gold substrate temperature was kept below $40^{\circ} \mathrm{C}$. The sample was cooled down with liquid helium before its transfer to the STM chamber. An aluminum source mounted in the STM chamber was used to deposit Al atoms on the gold surface. During this deposition, the sample was kept at cryogenic temperatures. All STM imaging, tunneling spectroscopy measurements and molecule manipulations were done at $\sim 7 \mathrm{~K}$ using a Createc LT-UHV-STM.

The VASP $\operatorname{code}^{15}$ was used to evaluate the geometry and electronic structure of the H-molecule adsorbed on $\mathrm{Au}(111)$ in the presence of $\mathrm{Al}$ and $\mathrm{Au}$ ad-atoms. The calculations were performed using the PBE generalized-gradient approximation. The plane wave basis set was converged to 
$400 \mathrm{eV}$ of cutoff energy and the k-point sampling was $7 \mathrm{x} 7 \mathrm{x} 1$ for an orthogonal $7 \mathrm{x} 4 \sqrt{3}$ unit cell of the $\mathrm{Au}$ (111). This $7 \mathrm{x} 4 \sqrt{ } 3$ unit cell is an almost square cell of $(20.36 \times 20.15) \AA$. The PBE functional was completed by semi-empirical van-der-Waals interactions as parametrized by Thatchenko and Scheffler. ${ }^{16}$

\section{ASSOCIATED CONTENT}

The Supporting Information is available free of charge on the ACS Publications website.

The Frota fitting of the STS Kondo resonance and the theoretically observed minimum energy conrigurations (PDF)

\section{AUTHOR INFORMATION}

\section{Corresponding Author}

*E-mail: we-hyo.soe@cemes.fr

\section{Author contributions}

W.-H.S. was responsible for planning and conducting the whole STM experiments with C.M. R.R. and N.L. were responsible for the calculations and the interpretation of the experiments with C.J.

\section{Notes}

The authors declare no competing financial interest.

\section{Acknowledgement}

We thank Prof. Antonio M. Echavarren (Institute of Chemical Research of Catalonia, Spain) and Prof. Claudia de León (Universidad Mariano Gálvez, Guatemala) for having kindly provided the 
tetrabenzo[a,c,j,h]phenazine molecule. This work has been supported by the AtMol European Commission Integrated Project and the Agency of Science, Technology and Research (A*STAR), Singapore - the Visiting Investigatorship Program: AtomTech Project (Phase III).

\section{REFERENCES}

(1) Kondo, J. Resistance Minimum in Dilute Magnetic Alloys, Prog. Theor. Phys. 1964, 32, $37-$ 49.

(2) Christou, G.; Gatteschi, D.; Hendrickson, D. N.; Sessoli, R. Single-Molecule Magnets, MRS Bulletin 2000, 25, 66-71.

(3) Madhavan, V.; Chen, V.; Jamneala, T.; Crommie, M. F.; Wingreen, N. S. Tunneling into a Single Magnetic Atom: Spectroscopic Evidence of the Kondo Resonance, Science 1998, $280,567-569$.

(4) Liang, W.; Shores, M. P.; Bockrath, M.; Long, J. R.; Park, H. Kondo Resonance in a SingleMolecule Transistor, Nature 2002, 417, 725-729.

(5) Temirov, R.; Lassise, A.; Anders, F.B.; Tautz, F.S. Kondo Effect by Controlled Cleavage of a Single-Molecule Contact, Nanotechnology 2008, 19, 065401.

(6) Zhao, A.; Li, Q.; Chen, L.; Xiang, H.; Wang, W.; Pan, S.; Wang, B.; Xiao, X.; Yang, J.; Hou, J. G.; Zhu, Q. Controlling the Kondo Effect of an Adsorbed Magnetic Ion Through Its Chemical Bonding, Science 2005, 309, 1542-1544.

(7) Komeda, T.; Isshiki, H.; Liu, J.; Zhang, Y. -F.; Lorente, N.; Katoh, K.; Breedlove, B. K.; Yamashita, M. Observation and Electric Current Control of a Local Spin in a SingleMolecule Magnet, Nat. Commun. 2011, 2, 217. 
(8) Ormaza, M.; Abufager, P.; Verlhac, B.; Bachellier, N.; Bocquet, M.L.; Lorente, N.; Limot, L. Controlled Spin Switching in a Metallocene Molecular Junction, Nat. Commun. 2017, 8, 1974.

(9) Krull, C.; Robles, R.; Mugarza, A.; Gambardella, P. Site- and Orbital-Dependent Charge Donation and Spin Manipulation in Electron-Doped Metal Phthalocyanines, Nat. Mater. 2013, 12, 337-343.

(10) Limot, L.; Pehlke, E.; Kröger, J.; Berndt, R. Surface-State Localization at Adatoms, Phys. Rev. Lett. 2005, 94, 036805.

(11) Frota, H. O.: Oliveira, L. N. Photoemission Spectroscopy for the Spin-Degenerate Anderson Model, Phys. Rev. B 1986, 33, 7871-7874(R).

(12) Uhl, W. Advances in Organometallic Chemistry 2004, 51, pp. 53-108.

(13) Ogrin, D.; van Poppel, L.H.; Bott, S.G.; Barron, A.R. Aluminium Alkyl and Aryloxide Complexes of Pyrazine and Bipyridines: Synthesis and Structure, Dalton Trans. 2004, 21, 3689-3694.

(14) Soe, W.-H.; Manzano, C.; De Sarkar, A.; Chandrasekhar, N.; Joachim, C. Direct Observation of Molecular Orbitals of Pentacene Physisorbed on Au(111) by Scanning Tunneling Microscope, Phys. Rev. Lett. 2009, 102, 176102.

(15) Kresse, G.; Hafner, J. Ab Initio Molecular Dynamics for Liquid Metals, Phys. Rev. B 1993, 47, 558-561(R) and Kresse, G.; Furthmüller, J. Efficiency of Ab-Initio Total Energy Calculations for Metals and Semiconductors Using a Plane-Wave Basis Set, Comput. Mater. Sci. 1996, 6, 15-50. 
(16) Tkatchenko, A.; Scheffler, M. Accurate Molecular Van Der Waals Interactions from Ground-State Electron Density and Free-Atom Reference Data, Phys. Rev. Lett. 2009, 102, 073005. 


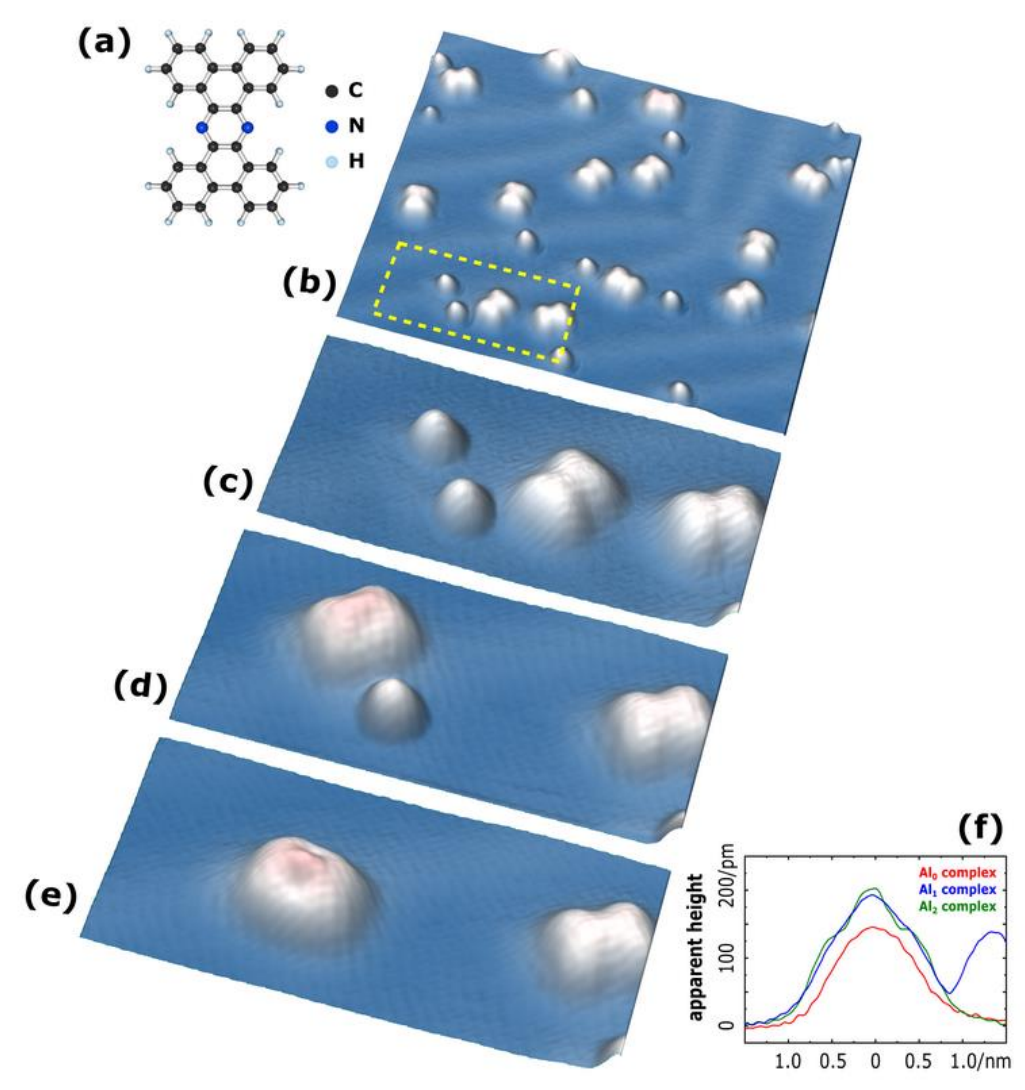

Figure 1. Construction of the aluminum metal complexes based on a tetrabenzo[a,c,j,h]phenazine molecule. (a) the molecule structure is presented by a ball and stick model. (b) An STM image taken after deposition of both ' $\mathrm{H}$ ' shape tetrabenzo[a,c,j,h]phenazine molecules and $\mathrm{Al}$ atoms. Image size is $20 \mathrm{~nm} \times 16 \mathrm{~nm}$ and bias voltage and tunneling current were set as $500 \mathrm{mV}$ and 30 pA. (c)-(e) A sequence of the zoomed-in images constructing the metal complexes by STM molecule manipulation technique. From (c) to (e), the STM junction tunnel resistance set up was between 1 to $2 \mathrm{M} \Omega$ to manipulate the molecule. Others manipulations sequences are presented in Supp. Info. S4. After the metal-molecule complex formation, a protrusion emerges at the center of molecule instead of the $\mathrm{Al}$ ad-atom features. Apparent height of center protrusion is slightly higher and sharper for the $\mathrm{Al}_{2}$-complex than for an $\mathrm{Al}_{1}$-complex as shown in cross-section profiles (f) recorded while scanning the STM tip apex across the molecule (red across (c), blue across (d) and green across (e)). 

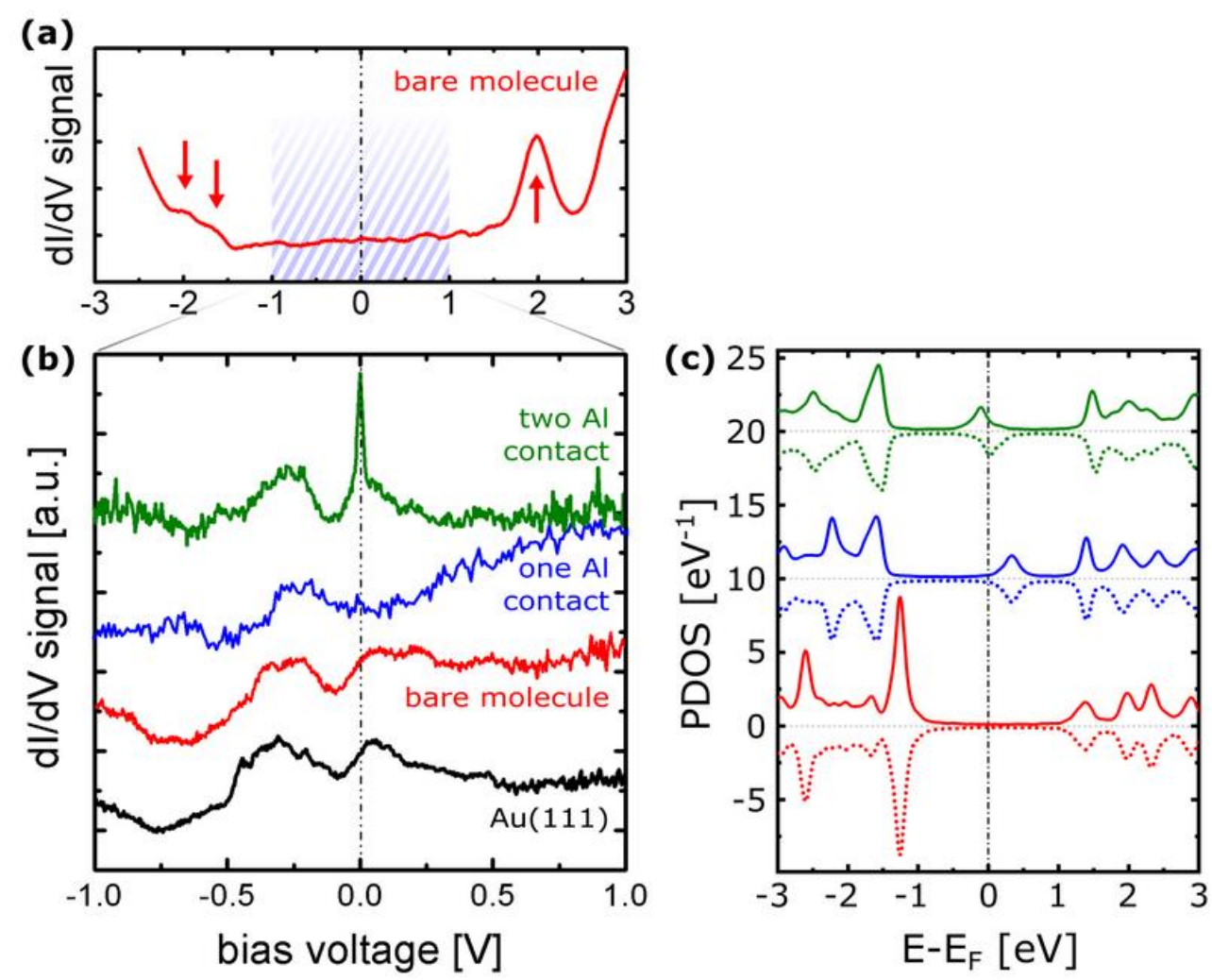

Figure 2. scanning tunneling spectra observed on the tetrabenzo[a,c,j,h]phenazine molecule and its metal complexes. (a) The spectrum taken on a bare $\mathrm{H}$ shaped molecule on the $\mathrm{Au}(111)$ surface with a wide bias voltage range. Two peaks are distinguished for each polarity corresponding to the first and second reduced states (positive) and the first and second oxidation states (negative) respectively. (b) Spectra obtained in the gap of the Al-complex molecular electronic states. The STM-bare molecule junction is showing no resonances. The spectrum from the Al1-molecule complex shows relatively higher differential conductance according to the voltage increase (blue). This is a tail of the first reduced state of this complex. Only in case of two Al atoms interacting with the molecule, a new sharp peak appears at a zero bias voltage. (c) The respective SCF-DFT density of states projected on the molecule for the 3 cases. Line colors are corresponding to experimentally recorded (b) dI/dV spectra. Experimental and calculated Data are shifted vertically for clarity. Solid- and dotted lines are corresponding to the up- and down-spins respectively. 

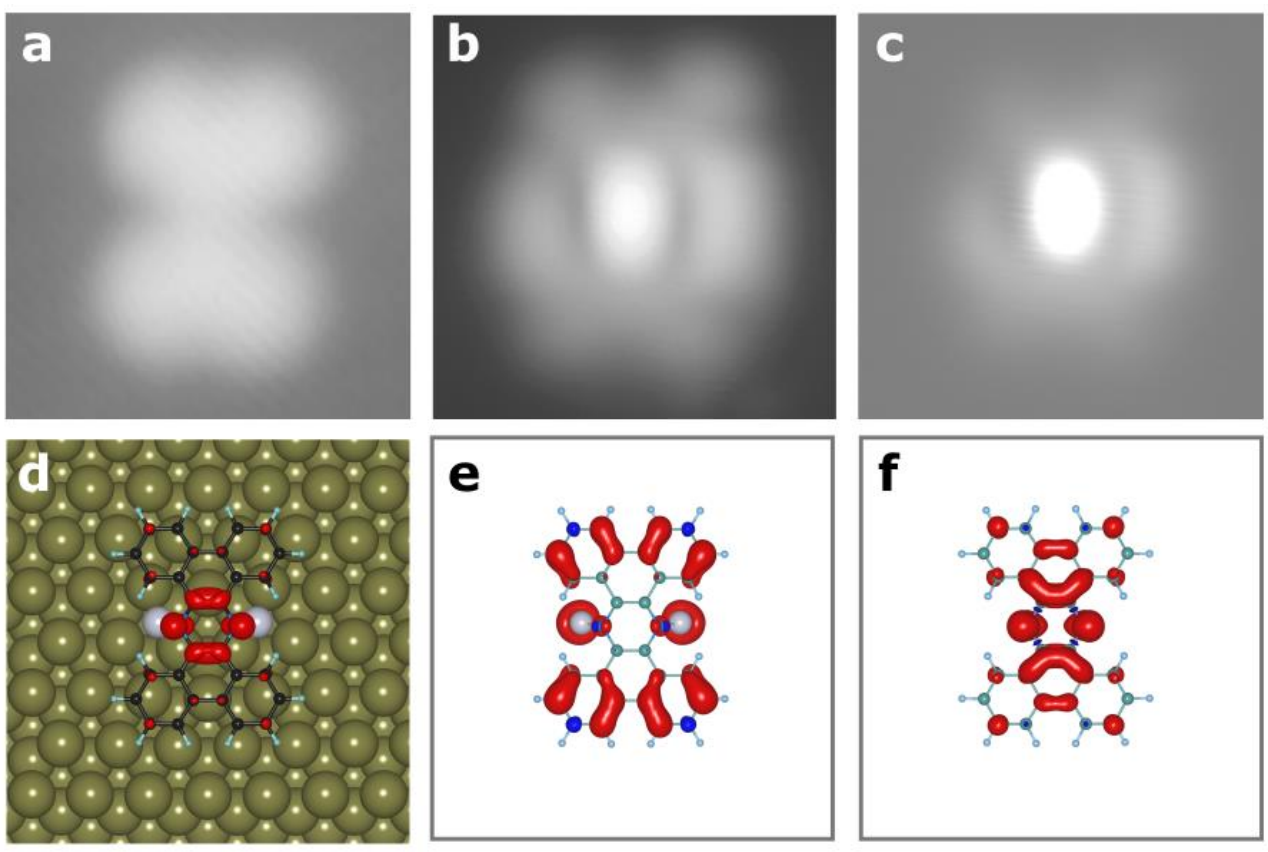

Figure 3. Experimental conductance maps vs. theoretical spin density map. STM constant current topographic images of (a) the bare tetrabenzo[a,c,j,h]phenazine molecule at $100 \mathrm{mV}$ as a reference and (b) $\mathrm{Al}_{2}$-molecule complex at $5 \mathrm{mV}$. (c) STS constant height differential conductance map at 5 $\mathrm{mV}$ using $1 \mathrm{mV}$ of modulation, and (d) spin density distribution calculated by DFT. $5 \mathrm{mV}$ of bias voltage was chosen to avoid lateral displacement of molecule during imaging due to tip-molecule interaction. The constant height mode was selected because $5 \mathrm{mV}$ is at the foot of the zero bias peak and a constant current differential conductance map at this voltage gave an indirect information about molecular states. ${ }^{14}$ Localizing spin density to the pyrazine part of the molecule creates the Kondo resonance due to the coupling with the $\mathrm{Au}(111)$ conduction electrons. (e) Spin density of the Al-molecule-Al system without $\mathrm{Au}(111)$ surface when three electrons have been removed to mimic charge transfer to the substrate cannot retrieve the adsorbed distribution. (f) The anion distribution of $\mathrm{H}$-molecule without $\mathrm{Al}$ atoms agrees with the distribution of the adsorbed system. This points at the decoupling of the molecule from the $\mathrm{Au}(111)$ substrate and $\mathrm{Al}$ ad-atoms. All image sizes are $2 \mathrm{~nm} \times 2 \mathrm{~nm}$. 